\title{
PRINCIPAIS MICOTOXICOSES EM SUÍNOS
}

\author{
Pollyana Cristina Maggio de Castro Souto ${ }^{1}$ \\ Laurinda Augusto ${ }^{1}$ \\ Mayra Carraro Di Gregorio ${ }^{1}$ \\ Carlos Augusto Fernandes de Oliveira ${ }^{2}$
}

\begin{abstract}
RESUMO
Diversas micotoxinas amplamente distribuídas na natureza possuem propriedades tóxicas acentuadas e podem contaminar culturas de milho destinadas à alimentação humana e animal. A aflatoxina e as fusariotoxinas (principalmente fumonisina e zearalenona) são as que mais comumente provocam prejuízos na suinocultura. A produção das fusariotoxinas ocorre principalmente antes da colheita, mas assim como a aflatoxina, também pode ocorrer póscolheita caso não seja tratada e seca corretamente. Os efeitos relacionados à ingestão destes compostos são dependentes de fatores relacionados à toxina (estrutura química, dose) e ao animal (espécies, raça, sexo, idade, condições nutricionais). Ainda, a possibilidade de ocorrência simultânea de duas ou mais micotoxinas, pode acarretar na potencialização de seus efeitos tóxicos sobre o organismo susceptível e, dependendo do grau de intoxicação, os sintomas desencadeados são representados principalmente pela baixa produtividade animal, maior susceptibilidade a doenças, o que gera grandes prejuízos econômicos. Esta revisão apresenta aspectos gerais relacionados à toxicologia, como mecanismos de ação e efeitos em suínos, além de dados de ocorrência de aflatoxinas, fumonisinas e zearalenona no milho brasileiro.
\end{abstract}

Palavras-chaves: micotoxinas, toxicidade, suínos.

\section{MAJOR MYCOTOXICOSES IN SWINE}

\begin{abstract}
Several mycotoxins widely distributed in nature have accentuated toxic properties and can contaminate corn crops for human and animal consumption. Aflatoxin and fusariotoxins (mainly zearalenone and fumonisin) are the most common causes of losses in pig farming. The production of fusariotoxins mainly occur before harvest, but just as aflatoxin, can also occur post-harvest if not treated and dried properly. The effects related to the ingestion of these compounds are dependent on factors related to the toxin (chemical structure, dose) and animal (species, breed, sex, age, nutritional conditions). Moreover, the possibility of simultaneous occurrence of two or more mycotoxins, may result in potentiation of their toxic effects on the organism susceptible and depending on the degree of intoxication, the triggered symptoms are primarily represented by the low animal productivity, increased susceptibility to diseases, which generates great economic losses. This review presents general aspects related to toxicology, as mechanisms of action and effects in pigs, as well as the occurrence of aflatoxin, fumonisin and zearalenone in Brazilian corn.
\end{abstract}

Keywords: mycotoxins, toxicity, swine.

\footnotetext{
${ }^{1}$ Doutorando em Zootecnia, Faculdade de Zootecnia e Engenharia de Alimentos, Universidade de São Paulo.

${ }^{2}$ Professor Titular de Micotoxicologia de Alimentos da Faculdade de Zootecnia e Engenharia de Alimentos da Universidade de São Paulo. Contato principal para correspondência. carlosaf@usp.br.
} 


\section{PRINCIPALES MYCOTOXICOSIS EN CERDOS}

\section{RESUMÉN}

Varias micotoxinas ampliamente distribuidas en la naturaleza tienen acentuadas propiedades tóxicas y pueden contaminar los cultivos de maíz para el consumo humano y animal. La aflatoxina y las fusariotoxinas (principalmente zearalenona y fumonisinas) son las que más comúnmente causan pérdidas en la cría de cerdos. La producción de fusariotoxinas ocurre principalmente antes de la cosecha, pero igual de aflatoxina, también puede ocurrir después de la cosecha si no se trata y se seca correctamente. Los efectos relacionados con la ingestión de estos compuestos dependen de factores relacionados con la toxina (estructura química, la dosis) y animal (especie, raza, sexo, edad, estado nutricional). Sin embargo, la posibilidad de ocurrencia simultánea de dos o más micotoxinas, puede dar lugar a la potenciación de sus efectos tóxicos sobre el organismo susceptible y, dependiendo del grado de intoxicación, los síntomas provocados están representados principalmente por la baja productividad de los animales, aumento de la susceptibilidad a las enfermedades, lo que genera grandes pérdidas económicas. Esta revisión presenta los aspectos generales relacionados con la toxicología, como mecanismos de acción y efectos en los cerdos, así como dados de ocurrencia de aflatoxina, zearalenona y fumonisinas en el maíz brasileño.

Palabras chave: micotoxinas, toxicidad, cerdos.

\section{INTRODUÇÃO}

Micotoxinas são metabólitos secundários tóxicos produzidos por fungos, que possuem estruturas químicas variadas e podem ser encontradas em diversos substratos destinados à alimentação humana e animal, principalmente no milho (1). A contaminação dos grãos por fungos toxigênicos pode ocorrer durante a produção, processamento, armazenamento ou estocagem (2). Mais de trezentas micotoxinas são conhecidas e produzidas por aproximadamente uma centena de fungos. As principais podem ser divididas em três grupos: aflatoxinas produzidas por fungos do gênero Aspergillus; ocratoxinas, produzidas por algumas espécies dos gêneros Aspergillus e Penicillium; e fusariotoxinas, que possuem como principais representantes a fumonisina, zearalenona e os tricotecenos, e são produzidas por diversas espécies do gênero Fusarium (1).

A diversidade de sintomas e a magnitude dos efeitos tóxicos observados (lesões de pele, hepatotoxicidade, nefrotoxicidade, neurotoxicidade, hematotoxicidade, genotoxicidade, mutagenicidade, teratogenicidade, imunossupressão e até mesmo a morte) estão relacionadas tanto às diferenças na estrutura química das micotoxinas, quanto pela variação individual do animal. Fatores como espécie, raça, sexo, idade, ambiente, manejo e condições nutricionais contribuem fortemente para esta variação $(3,4)$. As manifestações agudas de intoxicação ocorrem quando indivíduos consomem doses moderadas a altas de micotoxinas, enquanto que a ingestão prolongada de baixas doses de micotoxinas, conduz a uma toxicidade sub-crônica ou crônica. Adicionalmente, a possibilidade de ocorrência simultânea de duas ou mais micotoxinas, pode acarretar na potencialização dos efeitos tóxicos (4).

Atualmente o Brasil é responsável por $10 \%$ da exportação mundial de carne suína e, segundo a Associação Brasileira de Proteína Animal (5), a suinocultura cresce anualmente cerca de 4\%. Entretanto, condições climáticas ideais para o desenvolvimento de fungos toxigênicos são encontradas no Brasil e em outros países de clima tropical. As micotoxinas mais comumente encontradas e de maior relevância econômica são as aflatoxinas (AF), fumonisinas (FB) e zearalenona (ZEA). A ingestão destes compostos, principalmente pelo 
milho contaminado, gera grandes prejuízos econômicos pela redução do desempenho animal, da eficiência reprodutiva, da conversão alimentar, além de aumentar a susceptibilidade a doenças (4). A importância destas micotoxinas é relacionada não apenas pela ocorrência frequente, mas também pelo elevado potencial toxigênico demonstrado em animais de interesse zootécnico, principalmente suínos.

\section{AFLATOXINAS}

As aflatoxinas foram descobertas há mais de 50 anos, como a causadora da doença "X" dos perus na Inglaterra. Estudos laboratoriais subsequentes usando Aspergillus flavus confirmaram a existência de um princípio tóxico capaz de induzir toxicidade aguda no fígado. A elucidação estrutural da aflatoxina $\mathrm{B}_{1}\left(\mathrm{AFB}_{1}\right)$ foi realizada e confirmada pela sua síntese total em 1963 (6). As aflatoxinas são conhecidas por serem substâncias tóxicas, mutagênicas, teratogênicas, carcinogênicas e são apontadas como agentes causadores de câncer hepático e outros tipos de câncer em humanos (7).

A formação das aflatoxinas pode ocorrer na maioria dos substratos utilizados na alimentação humana e animal, principalmente o milho e amendoim, mas também em trigo, sementes de algodão, arroz, entre outros. Diversos compostos similares são conhecidos e designados pelo termo aflatoxina, entretanto, habitualmente este termo refere-se a quatro compostos de interesse médico-sanitário denominados $\mathrm{B}_{1}, \mathrm{~B}_{2}, \mathrm{G}_{1}$ e $\mathrm{G}_{2}$, além de dois produtos metabólicos - $\mathrm{M}_{1}$ e $\mathrm{M}_{2}(7,8)$. A estrutura química das aflatoxinas apresenta um núcleo central cumarínico ligado a uma estrutura bi-furanóide. As aflatoxinas B apresentam anel ciclopentona na molécula, enquanto que as da série $\mathrm{G}$ apresentam anel lactona (9).

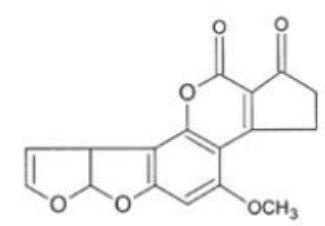

Aflatoxina B1

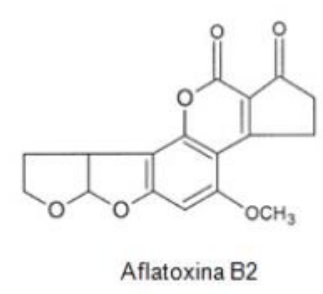

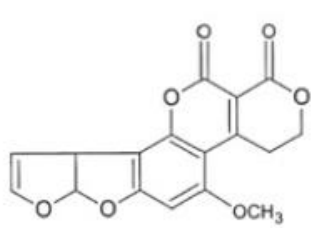

Aflatoxina G1

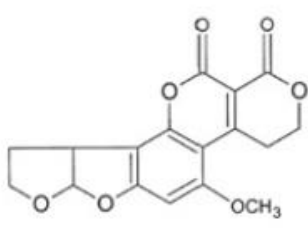

Aflatoxina G2

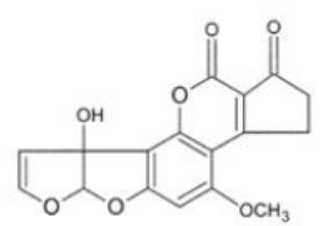

Aflatoxina M1

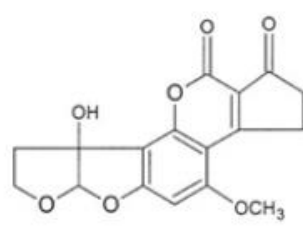

Aflatoxina M2

Figura 1. Estrutura química das aflatoxinas B1, G1, M1, B2, G2 e M2.

$A$ aflatoxina $B_{1}\left(A_{F B}\right)$, além de ser a mais frequentemente encontrada em substratos vegetais é a que apresenta maior poder toxigênico, enquanto que a toxicidade aguda das aflatoxinas $\mathrm{G}_{1}\left(\mathrm{AFG}_{1}\right), \mathrm{B}_{2}\left(\mathrm{AFB}_{2}\right)$ e $\mathrm{G}_{2}\left(\mathrm{AFG}_{2}\right)$ é cerca de 50,20 e $10 \%$ comparado à $\mathrm{AFB}_{1}$, respectivamente $(9)$.

\section{Mecanismo de ação das aflatoxinas}

Após a ingestão, a $\mathrm{AFB}_{1}$ é eficientemente absorvida por difusão passiva no trato gastrointestinal, rapidamente biotransformada no fígado antes de ser excretada. A AFB 1 absorvida e seus metabólitos são excretados pela urina, enquanto as fezes constituem uma via de eliminação para a $\mathrm{AFB}_{1}$ não absorvida e para os metabólitos liberados pela bile. No caso de 
lactantes, a excreção também pode ocorrer pelo leite. Estudos em animais demonstraram que em condições normais, $50 \%$ da dose oral de $\mathrm{AFB}_{1}$ administrada é rapidamente absorvida na região duodenal e alcança o fígado pelo sistema portal. $\mathrm{A} \mathrm{AFB}_{1}$ não metabolizada é concentrada no fígado, mas pode ser encontrada em quantidades bem menores nos rins e no sangue (7).

De forma geral, esta metabolização ocorre em duas fases conhecidas como Fase I e Fase II. A Fase I consiste principalmente por reações de hidrólise, redução e oxidação mediadas por enzimas, enquanto que a Fase II envolve as reações de conjugação do produto da Fase I. As enzimas da família do citocromo P450 (CYP) são as principais responsáveis pela biotransformação das aflatoxinas absorvidas (Fase I). Diversas enzimas CYP têm sido reportadas como envolvidas na biotransformação da $\mathrm{AFB}_{1}$ em 8,9-epóxido (AFBO). O AFBO é uma mistura de estereoisômeros $\mathrm{AFB}_{1}$-endo-8,9-epóxido (AFBO-endo) e $\mathrm{AFB}_{1}$-exo-8,9epóxido (AFBO-exo), sendo que apenas o último é um metabólito genotóxico. Apesar de AFBO-exo ter a meia-vida curta, são obtidos elevados rendimentos de adutos de DNA (10). A AFBO-exo pode ser covalentemente ligada ao DNA, formando os adutos $\mathrm{AFB}_{1}-\mathrm{N}^{7}$-guanina $\left(\mathrm{AFB}_{1}-\mathrm{N}^{7}\right.$-gua). A formação da $\mathrm{AFB}_{1}-\mathrm{N}^{7}$-gua ocorre pela ligação do $\mathrm{AFBO}$-exo com guaninas da molécula de DNA, na posição $\mathrm{N}^{7}$, ao nível do códon 249 do gene supressor de tumores p53. Esta ligação modifica a estrutura do DNA e, consequentemente, a sua atividade biológica, originando os mecanismos básicos de efeitos mutagênicos e carcinogênicos da $\mathrm{AFB}_{1}$. Esses adutos formados na molécula de DNA podem ser retirados da molécula após a sua formação, deixando sítios apurínicos na molécula de DNA, que tendem a ser preenchidos com adenina, resultando em transversão de guanina para timina, que promove a substituição de uma arginina por uma serina no produto proteico, um importante ponto de mutação (11).

A albumina é a única proteína plasmática identificada capaz de formar um aduto derivado da $\mathrm{AFB}_{1}$ - a $\mathrm{AFB}_{1}$-lisina ( $\mathrm{AFB}_{1}$-lys). Estes adutos de albumina podem ser formados a partir tanto do AFBO-exo quanto do AFBO-endo, ou ainda a partir de um produto de hidrólise do AFBO-exo - o diidrodiol - que é instável e sofre rearranjo catalisado por base para um dialdeído reativo (10).

$\mathrm{A} \mathrm{AFB}_{1}$ também pode ser metabolizada a vários outros compostos como as aflatoxinas $\mathrm{M}_{1}\left(\mathrm{AFM}_{1}\right), \mathrm{Q}_{1}\left(\mathrm{AFQ}_{1}\right), \mathrm{P}_{1}\left(\mathrm{AFP}_{1}\right), \mathrm{B}_{2 \mathrm{a}}\left(\mathrm{AFB}_{2 \mathrm{a}}\right)$ e uma espécie reduzida, o aflatoxicol (AFL). $\mathrm{AFM}_{1}$ é citotóxica, pode ser ativada para $\mathrm{AFM}_{1}-8,9$-epóxido e ligar-se ao DNA, sendo excretada na urina na forma de $\mathrm{AFM}_{1}$ ou $\mathrm{AFM}_{1}-\mathrm{N}^{7}$-guanina. $\mathrm{AFM}_{1}$ possui um potencial carcinogênico de $10 \%$ da $\mathrm{AFB}_{1} . \mathrm{AFM}_{1}$ é excretada pelo leite, urina e fezes (12). A $\mathrm{AFQ}_{1} \mathrm{e}$ $\mathrm{AFP}_{1}$ são considerados produtos de detoxificação. $\mathrm{A} \mathrm{AFB}_{2 \mathrm{a}}$ interfere diretamente na síntese de proteínas, levando a quadros de imunossupressão, interferência na coagulação sanguínea e às demais consequências das alterações provocadas por estas falhas no metabolismo (13). A formação de aflatoxicol é mediada por uma redutase citoplasmática, podendo ser reconvertido em $\mathrm{AFB}_{1}$ pela ação da desidrogenase. Assim, este metabólito é considerado como um reservatório de $\mathrm{AFB}_{1}$ em alguns organismos. O aflatoxicol apresenta cerca de $20 \%$ do potencial de mutagenicidade da $\mathrm{AFB}_{1}$ (14). As reações de Fase II são limitadas a conjugação do AFBO-exo com glutationa (GSH) catalisada pela enzima glutationa S-transferase (GST). Uma via alternativa para a desintoxicação AFBO é a sua hidrólise enzimática espontânea ou

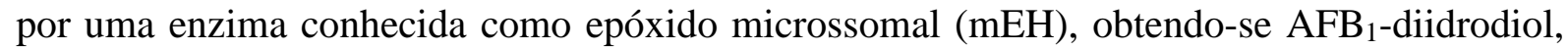
um metabolito menos tóxico (15).

Em suínos, apenas cerca de $4 \%$ da dose de $\mathrm{AFB}_{1}$ marcada (14C) recuperada nas fezes e na urina se encontravam nas formas de $\mathrm{AFB}_{1}$ inalterada e $\mathrm{AFM}_{1}$, com alguns traços de aflatoxicol. No plasma, a maior parte da radioatividade encontrada estava ligada às proteínas, com meia-vida biológica de aproximadamente 6 dias. Análises teciduais demonstraram que as atividades mais elevadas de $\mathrm{AFB}_{1}$ marcada foram nos dias 1 e 2 pós-exposição no fígado, no rim e pulmão (16). 


\section{Efeitos tóxicos das aflatoxinas em suínos}

A toxicidade das aflatoxinas está relacionada com a sua biotransformação pelo organismo tornando-as mais hidrofílicas a fim de que sejam excretadas facilmente. Assim, a $\mathrm{AFB}_{1}$ é considerada um pró-carcinógeno que requer uma ativação metabólica para manifestar seus efeitos tóxicos (17). Para muitas espécies os machos são mais susceptíveis do que as fêmeas e, em geral, a sensibilidade é acentuadamente maior nos jovens do que nos adultos (18). A intoxicação aguda por $\mathrm{AFB}_{1}$ caracteriza-se pela rápida deterioração do estado geral do animal, perda de apetite, hepatite aguda, icterícia, hemorragias e morte. O fígado apresenta lesões decorrentes de necrose hemorrágica, congestão centrolobular, proliferação das células dos ductos biliares e infiltração gordurosa dos hepatócitos $(18,19)$.

Os efeitos de toxicidade crônica são relacionados à genotoxicidade, lesões hepáticas (em menor escala quando comparadas à intoxicação aguda) e a diminuição do desempenho (consumo de ração e ganho de peso) (20). A disfunção hepática é observada principalmente pelas alterações nos níveis séricos de aspartato aminotransferase (AST), fosfatase alcalina (ALP), gama glutamiltransferase (GGT), albumina e proteína. Marin et al. (21) avaliaram o efeito da exposição de leitões desmamados à baixas doses de AF (140 e 280 ppb) por 4 semanas, sobre parâmetros hematológicos e imunológicos. O número total de leucócitos diminuiu em baixa dose de AF (140 ppb), enquanto que em dose elevada (280 ppb) teve o efeito oposto.

Consumo de AF também elevou a concentração de $\gamma$-globulina. A expressão de mRNA de citocinas em células sanguíneas indicou que a AF diminuiu a expressão de IL- $1 \beta$ e TNF- $\alpha$, e aumentou de IL-10, indicando que baixas doses de AF alteram vários aspectos da imunidade humoral e celular em suínos. Meissonier et al. (22) compararam a exposição subcrônica à $\mathrm{AFB}_{1}$ sobre as enzimas CYP e GST hepáticas em suínos expostos à 385, 867 ou 1807 ppb por 4 semanas. Os animais expostos à dose mais elevada de toxina desenvolveram sinais clínicos de aflatoxicose, como a fibrose hepática, aumento nos valores de ALP e GGT, e diminuição nos valores de proteína e albumina plasmáticas, e no ganho de peso. Este grupo tinha níveis hepáticos significativamente mais baixos de CYP e expressão proteíca de CYP1A e CYP3A. Os animais expostos à 867 ppb apresentaram alterações hepáticas degenerativas moderadas, sem disfunção hepática, mas um padrão semelhante de atividade hepática de enzimas CYP sem alteração na expressão CYP3A. Os animais expostos a 385 ppb de $\mathrm{AFB}_{1}$ apresentaram parâmetros semelhantes aos animais controle. Não foram observadas diferenças na atividade da GST entre os grupos experimentais. Assim, a exposição dietética subcrônica a baixos níveis de $\mathrm{AFB}_{1}$ não pode ser associada com sinais clínicos.

\section{FUMONISINAS}

Fusarium verticillioides (anteriormente $F$. moniliforme) é um dos fungos mais prevalentes associados à contaminação de milho em todo o mundo. Em 1970, esta linhagem foi isolada a partir de um lote de milho embolorado e acreditava-se ser o agente causador de um surto de uma doença denominada leucoencefalomalácia equina no Sul da África e do edema pulmonar suíno $(23,24)$. Posteriormente, o fungo foi extensivamente estudado, e, em 1988, as micotoxinas nomeadas fumonisinas foram isoladas em primeiro lugar a partir de culturas de $F$. verticillioides linhagem MRC 826. No mesmo ano, as estruturas químicas das fumonisinas também foram classificadas (25). Além $F$. verticillioides, que é a principal espécie produtora de fumonisinas, estas micotoxinas também podem ser produzidas por $F$. proliferatum, $F$. anthophilum, $F$. dlamini, F. napiforme e Alternaria alternata (26,27). Pelo menos 15 diferentes estruturas moleculares designadas pelo termo fumonisinas foram 
agrupadas em quatro principais categorias $(\mathrm{A}, \mathrm{B}, \mathrm{C}$ e $\mathrm{P})$, porém a toxina predominante produzida por linhagens de $F$. verticillioides é a fumonisina $\mathrm{B}_{1}\left(\mathrm{FB}_{1}\right)$. Em condições naturais, foram detectadas a produção apenas de $\mathrm{FB}_{1}$, fumonisina $\mathrm{B}_{2}\left(\mathrm{FB}_{2}\right)$ e fumonisina $\mathrm{B}_{3}\left(\mathrm{FB}_{3}\right)$ (27). A $\mathrm{FB}_{1}$ é a mais estudada e possui fórmula empírica $\mathrm{C}_{34} \mathrm{H}_{59} \mathrm{NO}_{15}$ composta por um diéster propano 1,2,3 - ácido tricarbalítico e 2-amino-12,16 dimetil-3,5,10,14,15-pentahidroxicosano em que nos C14 e C15 são esterificados com o grupo carboxiterminal de propano 1,2,3-ácido tricarbalítico $(28,29)$. Uma vez que as estruturas moleculares de $\mathrm{FB}_{2}$ e $\mathrm{FB}_{3}$ são quase idênticas a $\mathrm{FB}_{1}$, tem sido afirmado que a toxicidade destes compostos pode ser equivalente ao da $\mathrm{FB}_{1}$ (Figura 2) (25).

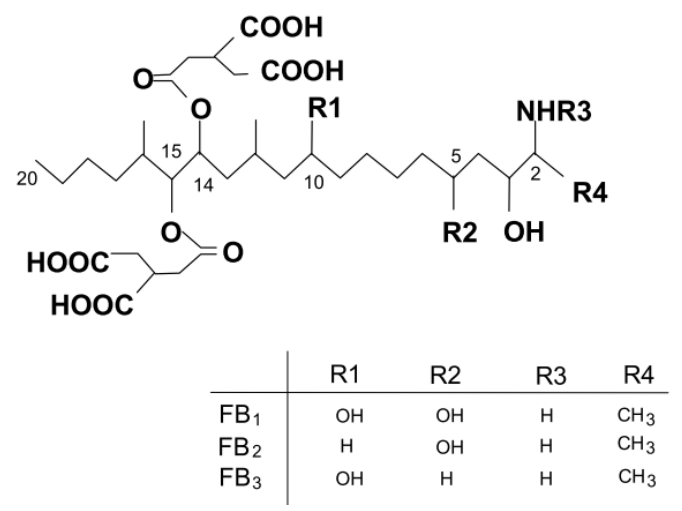

Figura 2. Estrutura molecular da fumonisina B1, B2 e B3.

Em contrapartida, os níveis de $\mathrm{FB}_{2}$ e $\mathrm{FB}_{3}$ comumente encontrados em alimentos são normalmente muito baixos (28). As moléculas pertencentes ao grupo fumonisina $\mathrm{B}\left(\mathrm{FB}_{1}, \mathrm{FB}_{2}\right.$, $\mathrm{FB}_{3}$ ), consistem de uma cadeia longa de hidrocarbonetos hidroxilados para que o ácido tricarbalílico, metilo e amino sejam adicionados.

Demonstrou-se que o grupo amino primário das fumonisinas é necessário para a atividade biológica de seus metabólitos, como a acetilação da $\mathrm{FB}_{1}$ para inibir a enzima cerimida sintase. Além disso, $\mathrm{FB}_{1}$ hidrolisada, a qual produz o ácido tricaborxílico e o aminopentol (AP-1) de $\mathrm{FB}_{1}$, representa $30-40 \%$ mais potente que $\mathrm{FB}_{1}$ intacta, destacando a importância das cadeias da molécula do ácido tricarbalítico.O aminopentol de FB $(\mathrm{AP}-1)$ é um inibidor e substrato para a enzima ceramida sintase, sendo a molécula resultante, $\mathrm{N}$ palmitoil-AP-1(PAP-1) um potente inibidor da enzima e dez vezes mais tóxica do que a $\mathrm{FB}_{1}$ intacta ou o metabólito AP-1 $(29,30)$.

\section{Mecanismo de ação das fumonisinas}

As fumonisinas são estruturalmente similares aos esfingolipídeos de membrana, os quais são importantes para a manutenção celular atuando como precursores de mensageiros secundários. Estes precursores modulam as respostas celulares aos fatores de crescimento e ativam uma cascata de enzimas que hidrolisam a esfingomielina em ceramida (esfingomielinases), ceramida em esfingosina 1-fosfato (esfingosina quinase) (30).

O mecanismo de ação das fumonisinas ainda não está completamente elucidado, o qual envolve muitos eventos bioquímicos. Contudo, Wang et al. (30) propuseram que a $\mathrm{FB}_{1}$ poderia intervir na biossíntese de esfingolipídeos ou na síntese "de novo" de esfingosina (Sa), devido a similaridade da molécula da cadeia longa encontrados nos esfingolipídeos de várias espécies. $\mathrm{O}$ sítio específico de ação de $\mathrm{FB}_{1}$ parece ser a inibição da enzima esfingosina $\mathrm{N}$ acetiltranferase (ceramida sintase). A inibição desta via metabólica resulta na depleção do complexo esfingolipídeo induzindo o aumento intracelular da concentração de Sa livre, ou, 
em menor grau, de esfingosina (So) livre levando a um aumento dos produtos de clivagem. $\mathrm{O}$ acúmulo de esfinganina livre, um composto altamente ativo, inicia uma cascata de alterações celulares que provavelmente é responsável pela causa primária da toxicidade da $\mathrm{FB}_{1}$ (31).

\section{Efeitos da fumonisina $B_{1}$ em suínos}

Estudos de toxicocinética indicam que a $\mathrm{FB}_{1}$ apresenta uma meia-vida muito curta em diferentes espécies de animais e consequentemente, baixa biodisponibilidade (28). A reduzida biodisponibilidade da $\mathrm{FB}_{1}$ deve-se, sobretudo, à sua fraca absorção (devido a sua hidrossolubilidade) e também à rápida eliminação pelo efeito de primeira passagem no fígado. Dentre os animais de produção, os suínos são os animais mais sensíveis às fumonisinas, sendo acometidos em níveis a partir de $0,2 \mathrm{mg} \mathrm{FB} / \mathrm{kg}$ corporal/dia (31).

$\mathrm{O}$ consumo de milho rico em $\mathrm{FB}_{1}$ induz ao aumento da expressão de proteínas citoprotetoras do trato gastrintestinal de suínos, especificamente na região do cólon (32). Do mesmo modo, outros efeitos biológicos da $\mathrm{FB}_{1}$, como alterações hepáticas, podem ser mensurados pelo aumento da atividade enzimática da fosfatase alcalina (ALP), sorbitol desidrogenase, aspartato aminotransferase (AST) e gama glutamiltransferase (GGT). As concentrações de colesterol sérico e ácidos biliares também apresentam elevações significantes, após a ingestão de rações contaminadas $(33,34)$. Estudos sugerem que o intestino é também um órgão-alvo para as fumonisinas $(35,36)$. Ambos os trabalhos encontraram um aumento da relação $\mathrm{Sa} / \mathrm{So}$ em soro de suínos alimentados com $\mathrm{FB}_{1}$ em concentrações $\geq 5 \mathrm{mg} / \mathrm{kg}$. Este aumento na proporção $\mathrm{Sa} / \mathrm{So}$ é considerado um biomarcador dose-dependente da exposição à fumonisina (25).

Os dados sobre o efeito das fumonisinas no desempenho animal descrevem que em níveis abaixo de $100 \mathrm{mg} / \mathrm{kg}$ na ração, o desempenho de suínos é pouco ou não alterado, enquanto que a ingestão de níveis mais elevados de $\mathrm{FB}_{1}$ prejudica consideravelmente o desempenho. Esta redução no desempenho geralmente é observada em suínos que apresentam sintomas de intoxicação ou lesões teciduais $(33,36)$. Embora as concentrações mais baixas de $\mathrm{FB}_{1}$ não afetem o desempenho animal, demonstrou-se que ingestão de $6,5-13 \mathrm{mg} / \mathrm{kg}$ de $\mathrm{FB}_{1}$ induz o aumento da colonização intestinal por patógenos como a Escherichia coli, além de modular negativamente a expressão gênica de mediadores imunológicos $(35,36)$.

O principal sintoma da toxicidade das fumonisinas em suínos foi denominado de edema pulmonar suíno (EPS) (24) após a eclosão de uma doença fatal em animais que consumiram milho contaminado com $F$. verticillioides provenientes da safra de grãos de 1989 em Iowa, Illinois e Georgia, nos Estados Unidos (23). Demonstrou-se nestes estudos, que a patogênese do edema pulmonar ou hidrotórax é decorrente da intoxicação aguda por $\mathrm{FB}_{1}$. A patogênese do edema pulmonar deve-se a danos no endotélio pulmonar, no epitélio alveolar e falência cardíaca, levando a óbito dentro de 4-7 dias de ingestão diária de fumonisinas em concentrações maiores ou iguais a $92 \mu \mathrm{g} / \mathrm{g}$ ou $6 \mathrm{mg} / \mathrm{kg}$ de peso corporal/dia. O mecanismo de indução do edema pulmonar por fumonisinas em suínos é atribuído a uma disfunção induzida no ventrículo esquerdo do coração ou, de modo diverso, devido ao aumento da permeabilidade vascular dos pulmões. Porém, o mecanismo está relacionado ao bloqueio da disponibilidade de íons $\mathrm{Ca}_{2}+$ tipo-L, mediado pelo esfingolipídeo denominado esfingosina, que foi alterada pelas fumonisinas. A ingestão de milho com fumonisinas em altas concentrações (112-400 mg/kg) provocou a morte de suínos por edema pulmonar agudo (24).

\section{ZEARALENONA}

Existem poucos registros sobre surtos com origem em contaminações com zearalenona. Em 1978 e 1981 em Porto Rico, e em 1997 no sudeste da Hungria, esta toxina foi considerada 
como agente causador de puberdade precoce em meninas (37). Inicialmente conhecida como toxina F-2, a zearalenona (ZEA) [6-(10-hidroxi-6-oxo-trans-1-undecenil)] é uma lactona do ácido beta-resorcílico, produzida por várias espécies de Fusarium, principalmente Fusarium graminearum, mas também por $F$. culmorum, $F$. cerealis, $F$. equiseti e $F$. incarnatum. Geralmente, estas espécies de Fusarium são encontradas como contaminantes de milho, cevada, aveia, trigo, sorgo e arroz $(38,39)$, porém a sua presença também foi detectada em produtos de cereais como farinha, malte, soja e cerveja. A produção desta toxina ocorre predominantemente antes da colheita, mas pode ocorrer pós-colheita caso não seja tratada e seca corretamente (40). Além de ZEA, estas espécies de fungos acima descritas, podem produzir pequenas quantidades de metabólitos, sendo os derivados mais importantes o $\alpha$ zearalenol $(\alpha$-ZOL) e $\beta$-zearalenol ( $\beta$-ZOL) (41).

Tanto a ZEA como seus metabólitos são compostos estrogênicos associados aos distúrbios reprodutivos de animais de produção (40). Contudo, o potencial estrogênico de $\alpha$ zearalenol é maior do que a da ZEA intacta e do metabólito $\beta$-zearalenol, provavelmente devido a uma maior afinidade de ligação aos receptores de estrogênio $(39,41)$. ZEA é estável ao calor de até $150^{\circ} \mathrm{C}$, e sua degradação foi observada apenas a altas temperaturas ou em condições alcalinas (42).

\section{Mecanismo de ação da zearalenona}

Após a ingestão, a ZEA é rapidamente absorvida (absorção 80-85\%) e, assim como seus derivados, podem ser detectados na circulação cerca de 30 minutos após a sua ligação aos hormônios reprodutivos. As vias metabólicas e o padrão de ingestão da zearalenona explicam as diferenças no mecanismo de ação nas diferentes espécies animais (38). Duas vias principais de biotransformação são sugeridas: i) hidroxilação pelo $3 \alpha$ - e $3 \beta$ - hidroxiesteroide

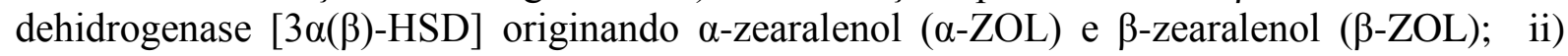
Conjugação de zearalenona e seus derivados com ácido glucorônico catalizado pela uridina difosfato glucuronil transferase, sendo o fígado o principal órgão onde ocorre a biotransformação (Figura 3) (43).

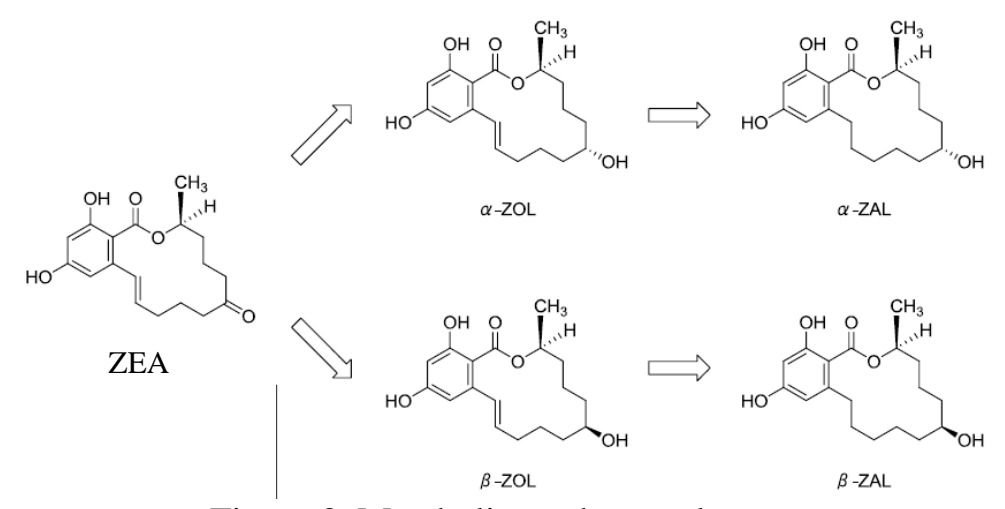

Figura 3. Metabolismo da zearalenona.

Em estudos realizados com suínos foram demonstrados uma alta atividade de conjugação glucurônica da mucosa intestinal comparada aos níveis de redução, sugerindo que a redução em metabólito zearalenol é antecedida pela conjugação, excreção biliar, reabsorção e redução (principalmente em $\alpha$-ZOL) pela mucosa intestinal e finalmente a entrada pela circulação via sistema porta pelo fígado (39). Segundo Minervini et al. (38), o ciclo enterohepático prolonga a retenção da zearalenona e seus derivados na circulação, retardando sua eliminação e aumentando a duração de seus efeitos adversos. Zearalenona e seus metabólitos ligam-se competitivamente aos receptores de estrogênio. Assim, a toxicidade está associada 
com distúrbios reprodutivos dependendo da espécie animal e, eventualmente, em seres humanos (41). Além da afinidade da zearalenona e seus metabólitos aos receptores de estrógeno, sua biotransformação em $\alpha$-ZOL e $\beta$-ZOL tem semelhanças aos processos do metabolismo dos esteróides sendo por isso também substrato da $3 \alpha(\beta)$-HSD presente em muitos tecidos como fígado, rins, testículos, próstata, hipotálamo, hipófise, ovário e intestino.

A conversão de testosterona e de progesterona por esses tecidos é um processo importante do mecanismo de modulação das gonadotrofinas e do comportamento sexual. Ao inibir competitivamente a redução desses hormônios, a zearalenona pode causar o acúmulo de compostos ativos com efeitos na reprodução, devido à afinidade aos receptores de estrógenos. No organismo animal, a degradação da zearalenona gera diversos metabólicos com maior ou menor estrogenicidade (38). A zearalenona e seus derivados competem efetivamente com o $17 \beta$-estradiol $\left(\mathrm{E}_{2}\right)$ por receptores específicos $(\alpha-\mathrm{e} \beta$-) em diferentes órgãos. Uma vez ligados aos receptores, a zearalenona e seus derivados iniciam uma sequência de eventos estimulados pelo estradiol em órgãos-alvo. A ligação da zearalenona aos receptores em tecidos alvo é < 1$10 \%$ comparada a de $\mathrm{E}_{2}$, enquanto que a ligação do $\alpha$-ZOL é maior e do $\beta$-ZOL é bem menor. No útero, o receptor citoplasmático é translocado para o núcleo, com efeito mais prolongado comparado a $\mathrm{E}_{2}$, induzindo eventos típicos de resposta antecipada a estrógeno como a síntese de RNA e aumento da atividade de RNA polimerase e síntese da proteína induzida pelo estrógeno e proliferação do endométrio (41).

\section{Efeitos tóxicos da zearalenona em suínos}

Em animais, os efeitos tóxicos da exposição prolongada com uma dieta contaminada com ZEA, incluem carcinogenicidade, genotoxicidade, toxicidade reprodutiva, efeitos endócrinos e imunotoxicidade, sendo os suínos pré-puberais um dos animais mais susceptíveis, com valores de $40 \mathrm{mg} / \mathrm{kg}$ de peso corporal/dia, em comparação a $100 \mathrm{mg} / \mathrm{kg}$ de peso corporal/dia em ratos. Assim, durante a gestação, as fêmeas contaminadas com ZEA apresentam redução da sobrevivência embrionária e fetal, apresentaram dilatação vulvar e vermelhidão, vulvovaginite, pseudogestação, retenção ou ausência de leite e prolapso retal. Nos machos, ZEA pode diminuir os níveis de testosterona, diminuição do peso dos testículos e espermatogênese, induzir a feminização e apresentar redução da libido (39).

Em estudos in vitro, Zhu et al. (44) relataram que ZEA em altas concentrações (30-120 $\mathrm{mM}$ ), diminui a proliferação de células granulosas de suínos, induzindo apoptose e necrose de modo dose-dependente. Adicionalmente, a ZEA induziu a perda do potencial mitocondrial de células da granulosa de suínos, aumentando os níveis de espécies reativas de oxigênio das células via caspase-3. Assim, estas evidências sugerem que ZEA ou seus metabólitos, $\alpha$-ZOL e $\beta$-ZOL, podem induzir atresia nos folículos ovarianos de suínos. Os efeitos dependem da dose consumida e da fase do ciclo estral ou de gestação em que ocorre o consumo de rações contaminadas com ZEA. A síndrome estrogênica em marrãs mais sensíveis que ingeriram entre $1-5 \mathrm{mg} / \mathrm{kg}$ da micotoxina, afeta primariamente o trato reprodutivo e a glândula mamária (39).

Em um estudo com leitoas pré-púberes alimentadas com $2 \mathrm{mg} / \mathrm{kg}$ de zearalenona durante 28 dias, Andretta et al. (45) observaram aumento significativo do tamanho e peso do trato reprodutivo, em particular da vulva. Em leitoas imaturas, a dose de $200 \mu \mathrm{g} / \mathrm{kg}$ de peso corporal induziu o desenvolvimento e maturação de folículos ovarianos pela ativação de um processo similar a apoptose na camada granulosa (44). Com relação aos oócitos de animais alimentados com ZEA e deoxinivalenol recuperados por ovariectomia, foram reportados folículos com cúmulos compactos e cromatina imatura resultando em maior proporção de oócitos com cromatina degenerada, porém não foi observada alteração na atividade das enzimas envolvidas na síntese de progesterona (46). 
A principal causa de esterilidade nestes animais é devida ao mau funcionamento do ovário, morte oocitária e anovulação mesmo com a manifestação do cio. A administração de 1 $\mathrm{mg} / \mathrm{kg}$ de peso corporal entre 7-10 dias depois da cobertura não interfere na gestação, porém quando administrado entre 2-5 dias leva a mudanças degenerativas no embrião que se evidenciam a partir do $13^{\circ}$ dia da gestação (46). Diaz-Llano e Smith (47) observaram aumento da incidência de filhotes mortos de marrãs gestantes alimentadas com grãos naturalmente contaminados com micotoxinas de Fusarium sp. Um estudo sobre o efeito da ZEA na maturação oocitária indicou que a exposição a $3,12 \mu \mathrm{mol} / \mathrm{L}$ afetou a maturação de $34 \%$ de oócitos e reduziu a quantidade dos que atingiram a fase de blastocisto para 12\% (48).

Em suínos machos expostos a elevados níveis de ZEA foram reportados uma redução de $30 \%$ no tamanho dos testículos e na fertilidade. Esta última está relacionada à redução na qualidade e viabilidade espermática, diminuição de níveis de testosterona e libido. Porém, em níveis normalmente presentes em alimentos usados na dieta animal não foram observadas alterações. A exposição de espermatozoides (in vitro) a zearalenona e seus derivados resultou em diferentes efeitos de cada composto. O $\alpha$-ZOL e a zearalenona a níveis de $\mathrm{pg} / \mathrm{mL}$ influenciaram negativamente a estabilidade da cromatina e a viabilidade espermática respectivamente, enquanto que o $\beta$-ZOL a níveis de $\mu \mathrm{g} / \mathrm{mL}$ influenciou negativamente a motilidade espermática (41).

\section{OCORRÊNCIA DE MICOTOXINAS EM MILHO NO BRASIL}

Embora diversos grãos (como sorgo, trigo e arroz) possam ser utilizados como ingredientes, a dieta de suínos é composta basicamente por milho (até $70 \%$ da formulação) e soja (49). Geralmente, o milho é cultivado em áreas de climas tropicais e subtropicais, com temperaturas e umidades variáveis, que favorecem o crescimento e a contaminação por diversos fungos produtores de micotoxinas. Estes eventos podem ocorrer em diversas fases do desenvolvimento, maturação, colheita, transporte, processamento ou armazenamento dos grãos (2).

Dentre os dados disponíveis (a partir do ano 2000) sobre a ocorrência natural de aflatoxinas, fumonisinas e zearalenona, na Tabela 1 estão apresentados alguns estudos realizados em milho e seus derivados no Brasil. Embora os dados reportados sejam amplamente variados, observa-se que tanto a frequência de contaminação, como os níveis encontrados são maiores para FB do que por AF e ZEA. Adicionalmente, não há no Brasil, até o momento, uma legislação estabelecendo limites de tolerância para micotoxinas em matérias primas e rações destinadas à alimentação de suínos ou outras espécies animais. 
Tabela 1. Níveis de aflatoxinas, fumonisinas e zearalenona detectados em amostras de milho e derivados no Brasil.

\begin{tabular}{lllll}
\hline Micotoxina & Estado de origem & $\begin{array}{l}\text { Frequência }^{\mathrm{a}} \\
(\%)\end{array}$ & $\begin{array}{l}\text { Concentração } \\
(\mu \mathrm{g} / \mathrm{kg})\end{array}$ & Referência \\
\hline Aflatoxinas & Bahia & 10,00 & $1-5$ & 50 \\
& Bahia & 8,00 & $13,7-47,8$ & 51 \\
& Mato Grosso & 18,00 & $6,8-976,1$ & 51 \\
& Rio Grande do Sul & 14,00 & $13,7-1393,0$ & 51 \\
& São Paulo & 2,00 & $2,0-34,2$ & 51 \\
& Mato Grosso & 23,80 & $1-108,7$ & 52 \\
& Paraná & 25,67 & $23,4-24,1$ & 53 \\
& Paraná & 9,33 & $35,2-40,0$ & 53 \\
& Goiás & 100,00 & $0,7-277,8$ & 54 \\
& Diversos & 38,00 & $0,4-139$ & 55 \\
\hline Fumonisinas & Diversos & 99,10 & $200-6.100$ & 55 \\
& Minas Gerais & 100,00 & $230-6.450$ & 56 \\
& São Paulo & $>90,00$ & $870-49.310$ & 57 \\
& São Paulo & 76,00 & $100-6.580$ & 58 \\
& Paraná & 100,00 & $32-6.000$ & 59 \\
& Paraná & 100,00 & $50-1.118$ & 59 \\
& Santa Catarina & 100,00 & $20-18.740$ & 60 \\
\hline Zearalenona & Minas Gerais & 98,00 & $1,8-99$ & 56 \\
& Paraná & 12,00 & nd-9,8 & 59 \\
& & 39,00 & nd-6,5 & 59 \\
\hline
\end{tabular}

\footnotetext{
${ }^{a}$ Número de amostras positivas/total de amostras analisadas.

${ }^{\mathrm{b}}$ Valores se referem às concentrações mínima e máxima. nd: não detectado.
}

\section{CONCLUSÃO}

O Brasil, devido ao seu clima típico, propicia condições ideais para a proliferação de fungos toxigênicos. Além disso, ainda prevalecem em diversas regiões do País, condições inadequadas de plantio, colheita, secagem, transporte e armazenamento de produtos agrícolas. Deste modo, a adoção de práticas agrícolas que previnam a contaminação e o desenvolvimento de fungos é fundamental para garantir a obtenção de insumos de boa qualidade para a elaboração de rações empregadas na suinocultura. Deve-se ressaltar que medidas que evitem a ocorrência de quadros de intoxicação por micotoxinas em suínos são fundamentais para a qualidade da carne produzida, o que impacta diretamente no lucro dos produtores, e em maior escala, na economia do País. Ainda, são necessários estudos sobre os mecanismos de ação das aflatoxinas, fumonisina e zearalenona em condições de exposição simultânea, mais prováveis de ocorrerem em condições de campo, com a finalidade de desenvolver alternativas efetivas para prevenir seus efeitos tóxicos em criações de suínos no Brasil.

\section{REFERÊNCIAS}

1. Diaz DE. The mycotoxin blue book. Nottingham: Nottingham University Press; 2005. 
2. Sabino M. Micotoxinas em alimentos. In: Oga S. Fundamentos de toxicologia. São Paulo: Atheneu; 1996. p.461-72.

3. Hussein HS, Brasel JM. Toxicity, metabolism and impact of mycotoxin on humans and animals. Toxicology. 2001;167:101-34.

4. Devreese M, Backer P, Croubels S. Overview of the most important mycotoxins for the pig and poultry husbandry. Vlaams Diergeneeskd Tijdschr. 2013;82,171-80.

5. Associação Brasileira de Proteína Animal. Exportação mundial de carne suína em 2014. São Paulo: ABPA; 2015. (Relatório Anual).

6. Wogan GN, Kensler TW, Groopman JD. Present and future directions of translational research on aflatoxin and hepatocellular carcinoma. A review. Food Addit Contam Part A Chem Anal Control Expo Risk Assess. 2012;29(2):249-57.

7. Jager AV, Ramalho FS, Ramalho LNZ, Oliveira CAF. Biomarkers of aflatoxin exposure and its relationship with the hepatocellular carcinoma. In: Guevara-Gonzalez RG. Aflatoxins - Biochemistry and molecular biology. Rijeka: Intech; 2011. p.104-26.

8. Coulombe RA. Aflatoxins. In: Sharma RP, Salunkhe DK. Mycotoxins and phytoalexins. Boca Raton: CRC Press; 1991. p.103-43.

9. Kussak A, Andersson B, Anderson K. Immunoafinity column clean-up for the high performance liquid choromatographic determination of aflatoxin B1, B2, G1, G2, M1 and Q1 in urine. J Chromatogr B Biomed Appl. 1995;672:253-59.

10. Guengerich FP. Cytochrome P450s and other enzymes in drug metabolism and toxicity. AAPS J. 2006;8:101-11.

11. Aguillar F, Hussain SP, Cerutti P. Aflatoxin B1 induces the transversion of G-T in códon 249 of the p53 tumor suppressor gene in human hepatocytes. Proc Natl Acad Sci U S A. 1993;90:8586-90.

12. Egner PA, Yu X, Johnson JK, Nathasingh CK, Groopman JD, Kensler TW, et al. Identification of aflatoxin M1-N7-guanine in liver and urine of tree shrews and rats following administration of aflatoxin B1. Chem Res Toxicol. 2003;16:1174-80.

13. Patterson DSP. Metabolism as a factor in determining the toxic action of the aflatoxins in different animal species. Food Cosmet Toxicol. 1973;11:287-94.

14. Lee S, Campbell BC, Molyneux RJ, Hasegawa S, Lee HS. Inhibitory effects of naturally occurring compounds on aflatoxin B1 biotransformation. J Agric Food Chem. 2001;49:5171-7.

15. Diaz GJ, Murcia HW. Biotransformation of Aflatoxin B1 and its relationship with the differential toxicological response to aflatoxin in commercial poultry species. In: Guevara-Gonzalez RG. Aflatoxins - Biochemistry and molecular biology. Rijeka: InTech; 2011. p.3-20.

16. Luthy J, Zweifel U, Schlatter C. Metabolism and tissue distribution of [C-14-labeled aflatoxin-B1 in pigs. Food Cosmet Toxicol. 1980;18:253-6.

17. Rawal S, Kim JE, Coulombe-JR R. Aflatoxin B1 in poultry: toxicology, metabolism and prevention. Res Vet Sci. 2010;89:325-31.

18. Leeson S, Diaz GJ, Summers JD. Poultry metabolic disorders and mycotoxins. Guelph: University Books; 1995. 
19. Osweiler GD. Mycotoxins and livestock: what role do fungal toxins play in illness and production losses? Vet Med. 1990;85:89-94.

20. Thieu NQ, Ogle B, Pettersson H. Efficacy of bentonite clay in ameliorating aflatoxicosis in piglets fed aflatoxin contaminated diets. Trop Anim Health Prod. 2008;40:649-56.

21. Marin DE, Taranu I, Bunaciu RP, Pascale F, Tudor DS, Avram N, et al. Changes in performance blood parameters, humoral and cellular immune responses in weanling piglets exposed to low doses of aflatoxina. J Anim Sci. 2002;80:1250-7.

22. Meissonnier GM, Laffitte J, Loiseau N, Benoit E, Raymond I, Pinton P, et al. Selective impairment of drug-metabolizing enzymes in pig liver during subchronic dietary exposure to aflatoxin B1. Food Chem Toxicol. 2007;45:2145-54.

23. Marasas WFO. Discovery and occurrence of the fumonisins: a historical perspective. Environ Health Perspect. 2001;109:239-43.

24. Haschek WM, Gumprecht LA, Smith G, Tumbleson ME, Constable PD. Fumonisin toxicosis in swine: an overview of porcine pulmonary edema and current perspectives. Environ Health Perspect. 2001;109:251-7.

25. Minami L, Meireles PG, Hirooka EY, Ono EYS. Fumonisinas: efeitos toxicológicos, mecanismo de ação e biomarcadores para avaliação da exposição. Cienc Agrar. 2004;25:207-24.

26. Chen J, Mirocha CJ, Xie W, Hogge L, Olson D. Production of the mycotoxin fumonisin B1 by Alternaria alternata f. sp. lycopersici. Appl Environ Microbiol. 1992;58:3928-31.

27. Ah-Seo J, Won Lee Y. Natural occurrence of the C series of fumonisins in moldy corn. Appl Environ Microbiol. 1999;65:1331-4.

28. Henry MH, Wyatt RD. A review of fumonisin production by Fusarium moniliforme and fumonisin toxicoses in animals. Appl Poult Res. 1993;2:188-92.

29. Soriano JM, González L, Catalá AI. Mechanism of action of sphingolipids and their metabolites in the toxicity of fumonisin B1. Prog Lipid Res. 2005;244:345-56.

30. Wang E, Norred WP, Bacon CW, Riley RT, Merrill Jr AH. Inhibition of sphingolipids biosynthesis by fumonisins. Implications for diseases associated with Fusarium moniliforme. J Biol Chem. 1991;266:14486-90.

31. Stockmann-Juvala H, Savolainen KA. A review of the toxic effects and mechanisms of action of fumonisin B1. Hum Exp Toxicol. 2008;27:799-809.

32. Lallès JP, Lessard M, Oswald IP, David JC. Consumption of fumonisin B1 for 9 days induces stress proteins along the gastrointestinal tract of pigs. Toxicon. 2010;55:244-9.

33. Dilkin P, Direito G, Simas MM, MallmanN CA, Corrêa B. Toxicokinetics and toxicological effects of single oral dose of fumonisin B1 containing Fusarium verticillioides culture material in weaned piglets. Chem Biol Interact. 2010;3:157-62.

34. Dilkin P, Zorzete P, Mallmann CA, Gomes JD, Utiyama CE, Oetting LL, et al. Toxicological effects of chronic low doses of aflatoxin B1 and fumonisin B1-containing Fusarium moniliforme culture material in weaned piglets. Food Chem Toxicol. 2003;41:1345-53.

35. Oswald IP, Desautels C, Laffitte J, Fournout S, Peres SY, Odin M, et al. Mycotoxin fumonisin B1 increases intestinal colonization by pathogenic Escherichia coli in pigs. Appl Environ Microbiol. 2003;69:5870-4. 
36. Bouhet S, Le Dorze E, Peres S, Fairbrother JM, Oswald I P. Mycotoxin fumonisin B1 selectively down-regulates the basal IL-8 expression in pig intestine: in vivo and in vitro studies. Food Chem Toxicol. 2006;44:1768-73.

37. Santos MC, Sousa RB, Oliveira SEM, Lima KSC, Lima ALS. Micotoxinas e seu potencial como agentes de guerra. Rev Virtual Quim. 2014;6:761-78.

38. Minervini F, Dell'Aquila ME. Zearalenone and reproductive function in farm animals. Int J Mol Sci. 2008;9:2570-84.

39. Zinedine A, Soriano JM, Moltó JC, Mañes J. Review on the toxicity, occurrence, metabolism, detoxification, regulations and intake of zearalenone: An oestrogenic mycotoxin. Food Chem Toxicol. 2007;45:1-18.

40. Richardson KE, Hagler WM, Mirocha CJ. Production of zearalenone, $\alpha$-zearalenol and $\beta$ zearalenol by Fusarium spp. in rice culture. J Agric Food Chem. 1985;33:862-6.

41. Fitzpatrick D, Picken CA, Murphy LC, Buhr MM. Measurement of the relative bindingaffinity of zearalenone, alpha-zearalenol and beta-zearalenol for uterine and oviduct estrogen-receptors in swine, rats and chickens: an indicator of estrogenic potencies. Comp Biochem Physiol. 1989;94:691-4.

42. Ryu D, Hanna MA, Bullerman LB. Stability of zearalenone during extrusion of corn grits. J Food Prot. 1999;62:1482-4.

43. Mizutani K, Nagatomi Y, Mochizuki N. Metabolism of zearalenone in the course of beer fermentation. Toxins. 2011;3:134-41.

44. Zhu L, Yuan H, Guo C, Lu Y, Deng S, Yang Y, et al. Zearalenone induces apoptosis and necrosis in porcine granulosa cells via a caspase-3- and caspase-9 dependent mitochondrial signaling pathway. J Cell Physiol. 2012;227:1814-20.

45. Andretta I, Lovatto PA, Hauschild L, Dilkin P, Garcia GG, Lanferdini E, et al. Feeding of pre-pubertal gilts with diets containing zearalenone. Arq Bras Med Vet Zootec. 2008;60:1227-33.

46. Malekinejad H, Schoevers EJ, Daemen IJJM, Zijlstra C, Colenbrander B, FinkGremmels J, et al. Exposure of oocytes to the Fusarium toxins zearalenone and deoxynivalenol causes aneuploidy and abnormal embryo development in pigs. Biol Reprod. 2007;77:840-7.

47. Diaz-llano G, Smith TK. Effects of feeding grains naturally contaminated with Fusarium mycotoxins with and without a polymeric glucomannan mycotoxin adsorbent on reproductive performance and serum chemistry of pregnant gilts. J Anim Sci. 2006;84:2361-6.

48. Alm H, Brüssow KP, Torner H, Vanselow J, Tomek W, Dänicke S, et al. Influence of Fusarium-toxin contaminated feed on initial quality and meiotic competence of gilt oocytes. Reprod Toxicol. 2006;22:44-50.

49. Rostagno HS, Albino LFT, Donzele JL, Gomes PC, Oliveira RF, Lopes DC, et al. Tabelas brasileiras para aves e suínos: composição de alimentos e exigências nutricionais. 3a ed. Viçosa: UFV; 2011.

50. Almeida AVAF, Botura MB, Abreu RD, Bittencourt TCC, Batatinha MJM. Ocorrência de aflatoxinas em milho destinado à alimentação de aves no estado da Bahia. Arq Inst Biol. 2009;76:353-8. 
51. Rocha LO, Nakai VK, Braghini R, Reis TA, Kobashigawa E, Corrêa B. Mycoflora and co-occurrence of fumonisins and aflatoxins in freshly harvested corn in different regions of Brazil. Int J Mol Sci. 2009;10:5090-103.

52. Bento LF, Caneppele MAB, Albuquerque MCF, Kobayasti L, Caneppele C, Andrade PJ, et al. Ocorrência de fungos e aflatoxinas em grãos de milho. Rev Inst Adolfo Lutz. 2012;71:44-9.

53. Moreno EC, Garcia GT, Ono MA, Vizoni E, Kawamura O, Hirooka EY, et al. Cooccurrence of mycotoxins in corn samples from the northern region of Paraná State, Brazil. Food Chem. 2009;116:220-6.

54. Ramos ATM. Levantamento da microflora de grãos ardidos de milho e avaliação da resistência genética à Fusarium verticillioides [dissertação]. Piracicaba: Escola Superior de Agricultura Luiz de Queiroz; 2008.

55. Vargas EA, Preis RA, Castro L, Silva CMG. Co-occurrence of aflatoxins B1, B2, G1, G2, zearalenone and fumonisin B1 in Brazilian corn. Food Addit Contam. 2001;18:9816.

56. Queiroz VAV, Alves GLO, Conceição RRP, Guimarães LJM, Mendes SM, Ribeiro PEA, et al. Occurrence of fumonisins and zearalenone in maize stored in family farm in Minas Gerais, Brazil. Food Control. 2012;28:83-6.

57. Orsi RB, Corrêa B, Pozzi CR, Schammas EA, Nogueira JR, Dias SMC, et al. Mycoflora and occurrence of fumonisins in freshly harvested and stored hybrid maize. J Stored Prod Res. 2000;36:75-87.

58. Camargos SM, Soares LMV, Sawazaki E, Bolonhezi D, Castro JL, Bortolleto N. Fumonisins in corn cultivars in the State of São Paulo. Braz J Microbiol. 2000;31:225-9.

59. Souza MLM, Sulyok M, Freitas-Silva O, Costa SS, Brabet C, Machinski Junior M, et al. Cooccurrence of mycotoxins in maize and poultry feeds from Brazil by liquid chromatography/tandem mass spectrometry. Sci World J. 2013:1-9.

60. Van Der Westhuizen L, Shephard GS, Scussel VM, Costa LLF, Vismer HF, Rheeder JP, et al. Fumonisin contamination and Fusarium incidence in corn from Santa Catarina, Brazil. J Agric Food Chem. 2003;51:5574-8.

Recebido em: 29/06/2016 Aceito em: 11/08/2017 